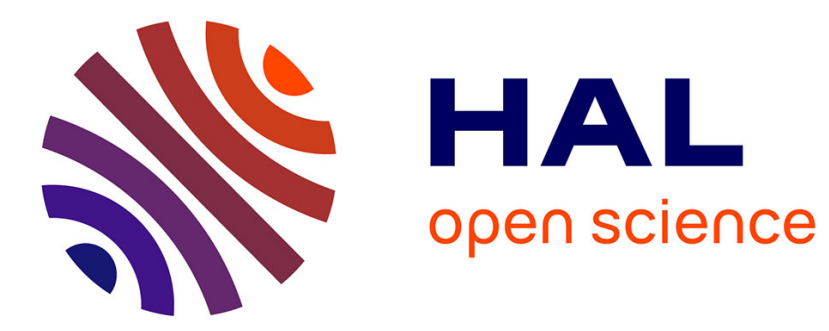

\title{
Expanding roles for AMPK in skeletal muscle plasticity
}

Rémi Mounier, Marine Théret, Louise Lantier, Marc Foretz, Benoit Viollet

\section{To cite this version:}

Rémi Mounier, Marine Théret, Louise Lantier, Marc Foretz, Benoit Viollet. Expanding roles for AMPK in skeletal muscle plasticity. Trends in Endocrinology and Metabolism = Trends in Endocrinology \& Metabolism , 2015, 26 (6), pp.275-286. 10.1016/j.tem.2015.02.009 . inserm-01171734

\section{HAL Id: inserm-01171734 https://www.hal.inserm.fr/inserm-01171734}

Submitted on 6 Jul 2015

HAL is a multi-disciplinary open access archive for the deposit and dissemination of scientific research documents, whether they are published or not. The documents may come from teaching and research institutions in France or abroad, or from public or private research centers.
L'archive ouverte pluridisciplinaire HAL, est destinée au dépôt et à la diffusion de documents scientifiques de niveau recherche, publiés ou non, émanant des établissements d'enseignement et de recherche français ou étrangers, des laboratoires publics ou privés. 
Expanding roles for AMPK in skeletal muscle plasticity

Rémi Mounier ${ }^{1,2}$, Marine Théret ${ }^{1,2,6}$, Louise Lantier ${ }^{3}$, Marc Foretz ${ }^{4,5,6}$ and Benoit Viollet ${ }^{4,5,6}$

${ }^{1}$ Centre de Génétique et de Physiologie Moléculaires et Cellulaires, UMR CNRS 5534, Villeurbanne, France

${ }^{2}$ Université Claude Bernard Lyon 1, Villeurbanne, France

${ }^{3}$ Vanderbilt University Medical Center, Molecular Physiology andBiophysics, Nashville, TN, USA

${ }^{4}$ INSERM, U1016, Institut Cochin, Paris, France

${ }^{5}$ CNRS, UMR8104, Paris, France

${ }^{6}$ Université Paris Descartes, Sorbonne Paris Cité, Paris, France

Corresponding author:Viollet, B. (benoit.viollet@inserm.fr) 


\begin{abstract}
Skeletal muscle possesses a remarkable plasticity and responds to environmental and physiological challenges by changing its phenotype in terms of size, composition,and metabolic properties. Muscle fibers rapidly adapt to drastic changes in energy demands during exercise through fine-tuning of the balance between catabolic and anabolic processes. One major sensor of energy demand in exercising muscle is AMP-activated protein kinase (AMPK). Recent advances have shed new light on the relevance of AMPKboth as a multitask gatekeeper and as an energy regulator in skeletal muscle. Here, we summarize recent findings on the function of AMPK in skeletal muscle adaptations to contraction andhighlightits role in the regulation of energy metabolism and the control of skeletal muscle regeneration post-injury.
\end{abstract}

\title{
Keywords
}

AMP-activated protein kinase; Exercise; Skeletal muscle; Regeneration;Muscular Diseases; Therapeutics. 


\section{Glossary}

Acetyl-coenzyme A (CoA) carboxylase (ACC): an enzyme that catalyzes the carboxylation of acetyl-CoA to malonyl-CoA, a metabolic intermediate that inhibits fatty acid uptake into mitochondria. ACC exists as two isoforms, ACC1 and ACC2. ACC1 is highly expressed in liver, and brown and white adipose and ACC2 is principally expressed in skeletal and cardiac muscles. ACC phosphorylation by AMPK leads to inhibition of its activity.

AMP-activated protein kinase (AMPK): a serine/threonine protein kinase and sensor of cellular energy and nutrient status in eukaryotic cells.

$\mathrm{Ca}^{2+} /$ calmodulin-dependent protein kinase kinase $\beta$ (CaMKK $\left.\beta\right)$ : a serine/threonine protein kinase that phosphorylates and activates AMPK when intracellular $\mathrm{Ca}^{2+}$ is increased.

Endurance exercise training: generally encompasses exercise durations of several minutes up to several hours at various exercise intensities, incorporating repetitive exercise such as cycling, running, or swimming.

Glucose transporter type 4 (GLUT4): an insulin-regulated glucose transporter primarily found in adipocytes, and cardiac and skeletal muscles. In skeletal muscle, insulin, contraction ,or AMPK activation induce the translocation of GLUT4 from intracellular vesicles to the plasma membrane for the delivery of glucose into the muscle cell.

Liver kinase B1 (LKB1): a serine/threonine protein kinase upstream of AMPK and other AMPK-relatedkinases. LKB1 activity does not change under conditions of metabolic stress such as during skeletal muscle contraction.

M1 pro-inflammatory macrophages: macrophages associated with acute inflammation that express specific pro-inflammatory cytokines such astumornecrosis factor $\alpha$ (TNF- $\alpha$ ) or IL$1 \beta \square$

M2 anti-inflammatory macrophages: macrophages associated with healing that express specific anti-inflammatory cytokines such as transforming growthfactor $\beta$ (TGF- $\beta$ ) or IL-10. 
Mechanistictarget of rapamycin complex (mTORC): composed of two distinct complexes mTORC1 and mTORC2, that are involved in protein synthesis, metabolism, proliferation, growth and survival. The mTORC and AMPK signaling pathways are opposite in their function.

Muscle glucose uptake: the mechanism by which glucose is transported from the plasma into the skeletal muscle cells via three serial steps: delivery of glucose from the blood to the sarcolemma, transport across the sarcolemmal membrane by GLUT; and intracellular phosphorylation to yield glucose6-phosphate, effectively trapping the glucose inside the muscle cell.

Resistance exercise training: generally encompasses short-duration activity at high or maximal exercise intensity, and increases the capacity to perform a single or relatively few repetitions of high-intensity exercise.

$\mathrm{NAD}^{+}$-dependent deacetylase silent mating-type information regulator2 homolog 1 (SIRT1):: an enzyme that deacetylates proteins that contribute tocellular regulation. SIRT1 acts as fuel sensor and plays a key role in theregulation of cellular stress responses..

Skeletal muscle: one of three types of muscle characterized by their connection to bone via tendons that enable voluntary bodily movements. Skeletal muscle comprises elongated, multinucleated cells termed myofibers that comprise organized, repeating cylinders of myofibrils. These contain the basic contractile unit, termed the sarcomere, which contains the filament, actin, and the motor protein, myosin. Skeletal muscle can be broken down into two main subtypes: red, slow, oxidative, type I and white, fast, glycolytic, type II muscles.

Tre-2/BUB2/cdc 1 domain 1 and 4 (TBC1D) 1/4): members of the Rab GTPase activating proteins (Rab-GAP) family of proteins that arephosphorylated by Akt and AMPK in response to insulin, muscle contraction and AICAR. 
Unccoordinated (unc)-51-like kinase 1 (ULK1): a serine/threonineprotein kinase andhomolog of yeast Atg1 that plays a key role in the formation of the pre-autophagosome structures during autophagy induction. 


\section{Phenotypic plasticity of skeletal muscle}

\section{- Skeletal muscle adaptation to the functional demands of exercise}

Skeletal muscle(see glossary) is a dynamic tissue with considerable plasticity. This is well illustrated by the adaptive changes occurringin response to various external stimuli (contractile activity, loading conditions, substrate supply, hormonal profile, and environmental factors) to match structural, functional, and metabolic demands[1].It is well established that physical exercise is a potent stimulus for the adaptation processes,and is known to remodel skeletal muscle to better respond to future challenges[2, 3]. Skeletal muscle is able to rapidly adapt to exercise interventions, and demonstrates remarkable malleability by changing its metabolic and contractile properties. Resistance exercise stimulates muscle protein synthesis and leads to growth of muscle fibers and hypertrophy. Bycontrast, endurance exercise leads to qualitative changes of muscle tissue by promoting phenotypic adaptations characterized mainly by fiber type transformation and increases in structures supporting oxygen delivery and consumption (mitochondrial biogenesis and angiogenesis), but no growth. Type IIb and type IIx fibers depend mainly on glycolytic pathways for ATP production, whereas type IIa and type I fibers rely predominantly on oxidative pathways.

\section{- Regulation of skeletal muscle energy balance during exercise}

Skeletal muscle contraction is associated with a dramatic increase in energy turnover and introduces a major energetic challenge to the muscle fiber. Change in the energy charge of the skeletal muscle leads to the activation of the AMP-dependent protein kinase (AMPK). This serine/threonine kinase, evolutionarily conserved in all eukaryotes, acts as an intracellular fuel sensor sensitive to the relative changes in the cellular AMP:ATPratio and, to a lesser extent ADP:ATP ratio, which together signal a fall in cellular energy status[4]. AMPK functions as a signaling hub,coordinating anabolic and catabolic pathways to balance 
nutrient supply with energy demand at both the cellular and whole-body levels. Exercise and skeletal muscle contractionare powerful physiological activators of AMPK as reportedthoroughly in exercising human skeletal muscle and in isolated contracting rodent skeletal muscle[5]. Exercise-induced AMPK activation has been shown to have regulatory effects on selected skeletal muscle processes, such as glucose uptake, fatty acid oxidation, glycogen metabolism, and protein synthesis as well as mitochondrial biogenesisto promote an oxidative muscle phenotype[6, 7]. The action of AMPK is achieved through acute phosphorylation of key regulatory proteins in carbohydrate, lipid, and protein metabolism for short-term effects, as well as phosphorylation of transcription factors and co-activators for longer-term regulatory effects $[7,8]$.

\section{AMPK: a safeguard of skeletal muscle energy metabolism}

\section{- AMPK structure and activation mechanism}

AMPK exists as a heterotrimeric complex comprising a catalytic subunit $\alpha$, a scaffolding $\beta$ subunit, and a regulatory $\gamma$ subunit (Figure 1). In mammals, each of the subunits occurs as multiple isoforms $(\alpha 1, \alpha 2, \beta 1, \beta 2, \gamma 1, \gamma 2$, and $\gamma 3)$, encoded by separate genes,enabling the formation of a diverse collection of $\alpha \beta \gamma$ heterotrimer combinations. The mechanism of AMPK activation involves two steps:a reversible phosphorylation at a conserved residue (Thr172 in rat $\alpha 1 / \alpha 2)$ in the $\alpha$ subunit, and a stimulatory allosteric binding of AMP within the C-terminal region of the $\gamma$ subunit.The $\beta$ subunit contains a central carbohydrate-binding module that causes AMPK to associate with glycogen particles and has inhibitory effects on AMPK activity [9] that prominently operate through $\alpha 2$-containing complexes[10]. In skeletal muscle, the major upstream kinase phosphorylating $\alpha$ subunit Thr172 is liver kinase B1 (LKB1), as exercise-induced AMPK phosphorylation is prevented 
in mouse models lacking LKB1[11, 12]. LKB1 is constitutively active, providing a continuous basal level of AMPK phosphorylation. AMPK activation is further enhanced by conformational changes imposed by binding of AMP and/or ADP to the $\gamma$ subunit,which promotes phosphorylation of Thr172and providesprotection against dephosphorylationby protein phosphatases[13, 14](Figure 2). Of note, the nature of the $\gamma$ subunit may determine the degree of allosteric activation, as $\gamma 2$ complexes have greater AMP sensitivity than $\gamma 1$ subunits, whichhave greater AMP sensitivity than $\gamma 3$ subunits [15]. The combined effect of phosphorylation on Thr172 and allosteric regulation causes a >1000-fold increase in kinase activity, allowing high sensitivity in responses to small changes in cellular energy status [16]. The $\mathrm{Ca}^{2+} /$ calmodulin-dependent protein kinase kinase $\beta(\mathrm{CaMKK} \beta)$ has also been shown to phosphorylate and activate AMPK in response to an increase in intracellular $\mathrm{Ca}^{2+}$ concentration, independent of any change in cellular AMP:ATP or ADP:ATP ratios[17, 18]. CaMKK $\beta$ has been shown to activate AMPK during mildtetanic skeletal muscle contraction[19] and increase AMPK $\alpha 1$ activity in response to skeletal muscle overload in LKB1-deficient mice [20].

\section{- AMPK expression profile and regulation of activity}

In human skeletal muscle, only three complexes have been detected: $\alpha 2 \beta 2 \gamma 1$ (65\% of the total pool), $\alpha 2 \beta 2 \gamma 3$ (20\%), and $\alpha 1 \beta 2 \gamma 1$ (15\%) [21](Figure 3). The heterotrimer combination varies in mouse skeletal muscle, with the detection of five complexes, including $\alpha 1$ and $\alpha 2$-associated AMPK complexes with both $\beta 1$ and $\beta 2$ isoforms [22](Figure 3). It also appears that the expression pattern of AMPK isoforms varies between muscles types [22](Figure 3). Of interest, the $\gamma 3$ isoform seems to be exclusively expressed in the fasttwitch glycolytic extensor digitorum longus (EDL) mouse muscle and not in the slow-twitch oxidative soleus mouse muscle [23]. Interestingly, a mutation (R225W) occurring in the gene 
encoding for the AMPK $\gamma 3$ subunitPRKAG3has been reported in pigs[24] and humans[25], causing increased deposition of glycogen in skeletal muscle. Recent studies have also revealed that each heterotrimer combination displays a distinct activation profile in response to physical exercise in human skeletal muscle,with $\gamma 3$-containing complexes $(\alpha 2 \beta 2 \gamma 3$ heterotrimer comprising $20 \%$ of total AMPK pool) predominantly activated [21], and $\alpha 2 \beta 2 \gamma 1$ and $\alpha 1 \beta 2 \gamma 1$ heterotrimers(comprising $80 \%$ of total AMPK pool) unchanged or activated only after prolonged exercise [26].In addition, the specificity of $\alpha 2$-containing complexes to translocate to the nucleus in response to muscle contraction expand the consequences of AMPK activation on the transcriptional programs in exercised skeletal muscle[27]. Of note, $\alpha 1$-containing complexes are usually slightly activated during exercise compared witha2-containing complexes.An additional layer of complexity emerged from recent studiesshowing differential function of specific AMPK heterotrimers, as evidenced by a distinct phosphorylation signature in exercised human muscle [28]. Compartmentalization of AMPK complexes within the myofiber may provide cluesfor heterotrimer substrate selection and particular functional roles. Heterotrimer subcellular localization has been shown to be dependent on both myristoylation and phosphorylation of the $\beta$ subunit [29] and the $\gamma$ associated isoforms in the heterotrimer complex [30].

\section{- AMPK in the control of exercise-induced metabolic optimization}

The conversion of skeletal muscle fibers from the more glycolytic fiber (type IIb) to the more oxidative fibers (types IIa, IIx and I) iscritical to optimize and enhance energy production during endurance exercise. This is consistent with the increase in the ratio of type $\mathrm{IIa} / \mathrm{x}$ fibers in skeletal muscle from mice expressing an AMPK $\gamma 1$ gain-of-function mutant (R70Q) [31] and from ratschronically treated with the AMP mimetic agent and AMPK activator 5-aminoimidazole-4-carboxamide-1- $\beta$-D-ribofuranoside (AICAR)[32]. In addition, 
using a transgenic mouse model with chronic inhibition of AMPKa2 activity, Rockl et al. demonstrated that AMPK activationparticipates inthe fiber type IIb to IIa/x transition during physical exercise training[31]. Interestingly, activation of AMPK has been recently involved in the control of fiber type specification by folliculin-interacting protein 1 (FNIP1)[33]. Loss of FNIP1 increases AMPK activation in skeletal muscle and results in a shift in muscle fibers from type IIb glycolytic fibers to type I and type IIa oxidative fibers, in a peroxisome proliferator-activated receptor $\gamma$ co-activator-1 $\alpha$ (PGC-1 $\alpha)$-dependent manner. Clearly, the negative interaction between FNIP1 and AMPK is an important area for future research, particularly in understanding the regulation of metabolic adaptation in skeletal muscle. Nevertheless, the role of AMPK in mediating glycolytic-to-oxidative fiber type transformation remains ambiguous, as AMPK $\gamma 3$ gain-of-function (R225Q) mice[34] and AMPK $\beta 1 \beta 2$-deficient mice display normal fiber distribution [35], whereasa compensatory increase in the percentage of type I fibershas beenreported in muscle AMPK $\alpha 1 \alpha 2$-deficient mice[36]at rest.Future investigations are needed to confirm the role of AMPK in fiber type specification by monitoring fiber type adaptation in AMPK $\alpha 1 \alpha 2$ - and AMPK $\beta 1 \beta 2$-deficient mice following exercise training.

\section{- AMPK in the control of fatty acid oxidation}

A major catabolic pathway activated during exercise is fatty acid oxidation. Data from experiments using mouse models have provided compelling evidence forthe important role of AMPK in regulating fatty acid metabolism in skeletal muscle.It has been shown that contraction-induced and pharmacological activation of AMPK stimulates fatty acid oxidation in skeletal muscle[37-40]. Thiswas further supported by the increase in fatty acid oxidation in skeletal muscle from transgenic mice expressing a AMPK $\gamma 3$ gain-offunction (R225Q) mutant [23]. It was also recently established that AMPK stimulates fatty 
acid oxidation in skeletal muscle through thephosphorylation and reduction of the activity of acetyl-coenzyme A (CoA) carboxylase 2 (ACC2), the enzyme that catalyzes the carboxylation of acetyl-CoA to malonyl-CoA, a metabolic intermediate involved in theregulation offatty acid oxidation[41]. However, controversies exist about the role of AMPK in regulating fatty acid oxidation in skeletal muscle due to the dissociation between AMPK activation, ACC phosphorylation, and malonyl-CoA levels during prolonged exercise in humans [42], indicating that muscle lipid oxidation isnot completely dependent on the AMPK/ACC pathway. Similarly, studies from transgenic mouse models with altered AMPK activity in skeletal muscleled to the conclusions that AMPK is not involved in the regulation offatty acid oxidation during exercise [43-45], but the presence of residual ACC phosphorylation in the muscle of these animals may contribute to the normal metabolic adaptation to contraction and the contribution ofthe AMPK/ ACC pathway cannot be definitively excluded. Thus, further studies involving the use of knockout mouse models are warranted in order to fully understand the contribution of muscle AMPK in the induction of fatty acid oxidation during exercise.

Another mechanism to enhance fatty acid utilization involves the translocation of the fatty acid transporter cluster of differentiation 36 (CD36)to the plasma membrane, to regulate fatty acid import into muscle cells. Although previous studies indicated that AMPK was not essential in the regulation of CD36 translocation and fatty acid uptake during contraction[35, 46], recent genetic evidence demonstrated that muscle AMPK $\alpha 1 \alpha 2$-deficient mice have impaired fatty acid utilization during in vivo exercise[47].Absence of exerciseinduced S237 phosphorylation of the Rab GTPase activating proteinTre-2/BUB2/cdc 1 domain (TBC1D) 1 in AMPK $\alpha 1 \alpha 2$-deficient muscle may provide a molecular mechanism explaining the defect in CD36 translocation and altered fatty acid uptake in response to contraction[47] (Figure 4). 


\section{- AMPK in the regulation of exercise-induced glucose transport}

During exercise, contracting skeletal muscle rapidly increases glucose uptake in an intensity-dependent manner, to sustain the energy demand caused by increased ATP turnover. This increase in skeletal muscle glucose uptake is driven by increased capillary recruitment as well as translocation of glucose transporter type 4 (GLUT4) to the plasma membrane (Figure 4). This latter event is required for increased glucose uptake during exercise, in both humans and rodents. The mechanisms underlying contraction-induced stimulation of glucose uptake have garnered considerable interest, as skeletal muscle contraction is a more potent stimulus of skeletal muscle glucose uptake than maximal insulin, and the proximal molecular mechanisms regulating insulin and contraction-stimulated glucose transport are distinct [48].However, recent findings have shown a convergence of these signals at TBC1D1 and TBC1D4,which are emerging as essential players in bothinsulin- and contraction-stimulated glucose uptake in skeletal muscle[49-51].It is also important to note that, unlike insulindependent glucose uptake, AMPK-mediated glucose uptake is not impaired in type 2 diabetes during exercise[52] and therefore activation of AMPK represents an attractive target for intervention.Consistent with this notion, therapeutic doses of metformin increased AMPK activity in the skeletal muscle of type 2 diabetics, along with higher rates of glucose disposal [53].

In recent years, the importance of AMPK in skeletal muscle glucose uptake has been examined extensively in genetically modified mouse models. Studies using the AMPK activatorsAICAR $22,37,54,55]$ and ex229, a cyclic benzimidazole derivative and potent direct AMPK activator [56], have shown that pharmacological activation of AMPK is sufficient to cause an increase in glucose transport in resting rodent muscle. Intriguingly, although AMPK activation by the direct AMPK activator A-769662 is associated with 
increased glucose uptake in isolated mouse muscle, its action appears to be mediated through a phosphoinositide 3-kinase (PI3K)-dependent pathway, indicating potential off-target effects[22]. There is genetic evidence to support an important role for AMPK in regulating TBC1D1 and TBC1D4 phosphorylation in response to exercise[35, 57, 58]. Consistent with these results, AICAR-mediated glucose uptake is blunted in skeletal muscle from TBC1D1and TBC1D4-deficient mice[49-51].Interestingly, in exercised human skeletal muscle, TBC1D4 phosphorylation is significantly correlated with an increase in $\alpha 2 \beta 2 \gamma 1$ heterotrimer activation, whereas TBC1D1 phosphorylation is correlated with the activity of the $\alpha 2 \beta 2 \gamma 3$ heterotrimer[28].However, it remains unclear whether AMPK is necessary for contractionstimulated glucose transport, as various transgenic AMPK-deficient mouse models display decreased, normal, or increased glucose transport, depending on the study [35, 36]. Musclespecific knockout of both $\beta$-AMPK subunits inhibited contraction-stimulated glucose uptake [35], while muscle-specific AMPK- $\alpha$ deficient mice showed reduced contraction-stimulated glucose uptake in soleus but not EDL muscle [36]. These conflicting results could be due to redundancy of signaling or diversity in contraction protocols and genetic models, but also highlight the extremely complex regulation of contraction-induced glucose uptake. It seems unwise to expect a single pathway to account for the full control of contraction-induced muscle glucose uptake.Muscle contraction is a multifactorial process and potential signals include changes in cellular energy status, reduced creatine phosphate and glycogen levels,increases in sarcoplasmic $\mathrm{Ca}^{2+}$ levels,generation of reactive oxygen species, and altered redox state. Based on the finding that specific $\mathrm{Ca}^{2+}$-activated proteins are necessary for the translocation of vesicles to the plasma membrane, a direct stimulatory effect of $\mathrm{Ca}^{2+}$ release from the sarcoplasmic reticulum during contraction is believed to act as a direct feedforward mechanism to facilitate GLUT4-mediated glucose transport independently of AMPK activation [59].However, Jensen et al. have recently reported the contrastingfinding, that both 
AMPK and mechanical stress are required for increased glucose transport in skeletal muscle, and are sufficient to recruit the full contraction-sensitive glucose transport response, while sarcoplasmic $\mathrm{Ca}^{2+}$ release is neither sufficient nor required, challenging the working model whereby skeletal muscle glucose transport is directly stimulated by $\mathrm{Ca}^{2+}$ released from the sarcoplasmic reticulum [60].

\section{- AMPK-dependent long-term control of exercise-induced metabolic adaptations}

AMPK has emerged as a key mediator of the long-term adaptation to training,with the ability to regulate the transcription ofthe glucose transporter GLUT4 and genes involved in mitochondrial biogenesis[61](Figure 4). Enhanced GLUT4 gene expression is dependent onan AMPK-dependent phosphorylation of the transcriptional repressor histone deacetylase 5 (HDAC5)following exercise [8]. The transcriptional control of mitochondrial biogenesis is mediated through the regulation of PGC-1 $\alpha$, a transcriptional co-activator that promotes the expression of mitochondrial genes encoded in both nuclear and mitochondrial DNA. AMPK activation causes the stimulation of PGC-1 $\alpha$ by direct phosphorylation [62]but also involves deacetylation through the silent mating-type information regulator 2 homolog 1 (SIRT1), which is downstream of AMPK,for the triggering of exercise-induced skeletal muscle adaptations [63].AMPK also controls the expression of PGC-1 $\alpha$ by phosphorylating HDAC5, leading to the release of myocyte enhancer factor 2 (MEF2) from repression by the histone deacetylaseand the activation of PGC-1 $\alpha$ gene transcription [61, 64]. Interestingly,the regulation of HDAC5 phosphorylation can be maintained during exercise despite the absence of AMPK by a compensatory activation of protein kinase D (PKD), an alternative HDAC5 kinase, to control PGC-1 $\alpha$ gene expression [65]. These results highlight the importance of the adaptive response in maintaining the integrity and metabolic capacity of skeletal muscle. In the same line, PGC-1 $\alpha$-deficient mice remainable to adapt to exercise trainingin a normal 
fashion, suggesting that other, currently unidentified factors are implicated in exerciseinduced adaptations [66].

The regulation ofPGC-1 1 by AMPKis consistent with the critical role of AMPK in initiating mitochondrial biogenesis in response to increases in the intramuscular AMP:ATP ratio by the creatine analogue $\beta$-guanadinipropionic acid ( $\beta$-GPA) [67]. In addition, reduced muscle AMPK activity has been associated with decreased mitochondrial content during aging [68] and in skeletal muscle from AMPK $\beta 1 \beta 2$ - and LKB1-deficient mice [35, 69]. However, there are some controversies regarding the role of AMPKin muscle mitochondrial biogenesis, as data from AMPK $\alpha 1 \alpha 2$-deficient mice indicate thatAMPK is dispensable for the regulation of baseline mitochondrial muscle content[36]. Nevertheless,loss of AMPK activity alters skeletal muscle basal metabolic functionthrough the reduction inthe expression of mitochondrial proteins and mitochondrial respiratory capacity[36, 70, 71] and may contribute to the exercise intolerance observed in muscle-specific AMPK-deficient mice $[35,36,72]$.

\section{- $A M P K$ in the regulation of skeletal muscle exercise-induced autophagy}

Recent studies have documented the role of autophagy in the plasticity of skeletal muscle in response to exercise [73, 74]. Autophagy is the process by which organelles and macromolecular complexes are engulfed into double-membraned vesicles, called autophagosomes, and delivered to lysosomes for digestion. During contraction, activation of autophagy ensures optimal energy utilization for maintaining cellular energy homeostasis, as well as the efficient turnover of damaged organelles following strenuous physical activity. AMPK has been shown to play a fundamental role in the stimulation of autophagy in skeletal muscle cells by phosphorylating the transcription factor Forkhead box protein O3a (FoxO3a), and the autophagy-related protein uncoordinated(unc)-51-like kinase 1 (ULK1), which participates in the initiation of autophagosome formation [75]. It has been proposed that 
autophagy may function in a feed-forward manner to activate AMPK [74], but studies in mice deleted of the autophagy-related gene autophagy-related protein 7(atg7) specifically in skeletal muscle challenged this hypothesis. Lo Verso et al. showed that inhibition of autophagy has no impact on AMPK $\alpha 1$ activation and energy provision to fuel contraction[76]. However, autophagy appears to be critical for the maintenance of mitochondrial quality control during damaging physical activity[76].

\section{AMPK: a new player in skeletal muscle regeneration}

\section{- AMPK, inflammation, and skeletal muscle regeneration}

Adult skeletal muscle possesses aremarkable capacity to regenerate after injury, exercise, or disease. Recent studies revealed that skeletalmuscleregeneration(Box 1) requires the migration, proliferation, and fusion of myoblasts to form multinucleated myotubes as well as interaction with other cell types within the muscle, including immune cells[77-79]. The inflammatory response during skeletal muscle regeneration is a spatially and temporally coordinated process. While the first steps of the inflammatory response are associated with M1 macrophages, which secrete pro-inflammatory cytokines, resolution of inflammation is associated with M2 macrophages, which exhibitan anti-inflammatory phenotype. The inflammatory resolution is a critical step and its alteration impairs skeletal muscle regeneration. In parallel of its role as an energy sensor, AMPK activation is associated with a decrease in the inflammatory response. Onbacterial lipopolysaccharide (LPS) stimulation in vitro, while AMPK activation decreases the secretion of pro-inflammatory compounds by macrophages, blocking of AMPK action induces an enhanced pro-inflammatory response [8082]. In vivo, the administration of AICAR attenuates LPS-induced acute lung injury [82], whereas the inhibition of AMPK leads to the opposite effect [83]. 
Recent evidence demonstrated that macrophage AMPK $\alpha 1$ is crucial for the resolution of inflammation during skeletal muscle regeneration [84]. Specific deletion of AMPKalin macrophages induces a delay in macrophage skewing (M1-to-M2 transition),impairing skeletal muscle regeneration. Inversely, rescue experiments with wild type bone marrow transplantation in AMPK $\alpha 1$-deficient mice improve skeletal muscle regeneration. During the regeneration process, AMPK $\alpha 1$ activation and macrophage skewing are linked to the phagocytosis of apoptotic/necrotic myofibers. In this context, AMPK $\alpha 1$ activation in macrophagesis dependent on phosphorylation by CaMKK $\beta$ but not LKB1[84] (Figure 5).

\section{- Energy and muscle stem cell fate choice}

During post-natal skeletal muscle regeneration, muscle stem cells activate and recapitulate the myogenic program to repair damaged myofibers. Muscle stem cells are capable of both differentiation to repair muscle tissue after injury, and self-renewal to replenish the stem cell pool. Control of the return to quiescence is crucial for skeletal muscle homeostasis, in order to maintain the pool of satellite cells for future needs. Molecular mechanisms that control muscle stem cell self-renewal are starting to be understood and some factors involved in this process have now been identified $[85,86]$. Interestingly, the AMPK upstream kinase LKB1 was shown to limit satellite cell proliferation through the AMPK/ mechanistic target of rapamycin complex1 (mTORC1)pathway[87]. In addition, FoxO3, an AMPK-regulated transcription factor,has recentlybeen involved in muscle stem cell selfrenewal by pushing cells to quiescence [88].Moreover, it has been established that endothelial cells trigger expansion cues to muscle stem cellsthrough the release of vascular endothelial growth factor (VEGF), insulin-like growth factor-1 (IGF-1), andfibroblast growth factors (FGFs)[89], while perivascular cells promote muscle stem cells self-renewal and quiescence through the Angiopoietin-1/Tie-2/extracellular signal-relatedkinase (ERK)1/2 axis [90]. The 
observation of increased VEGF expression in LKB1-deficient mice[91] reinforces the putativelink between theLKB1/ AMPK pathway andthecontrol of muscle stem cell selfrenewal. However, further studies in mice lacking AMPK activity in vascular cells are needed to validate this hypothesis.

It has recently been claimed that regulation of stem cell fate depends on the metabolicstate of the cell and the surrounding stem cell niche[92, 93]. Recent work from Ryall et al.investigated how metabolic reprogramming promotes the activation of satellite cells and modulates myogenic cell fate[94]. Muscle stem cell activation is associated with an increase in glycolysis that induces a decrease in intracellular $\mathrm{NAD}^{+}$and a decrease in SIRT1 expression. This leads to an increase in acetylated H4K16, an epigenetic mark of transcriptional activity, and the derepression of several muscle developmental genes. These findings reveal a tight link between the metabolic state of satellite cells and epigenetic modifications that regulate their myogenic commitment. Thus, manipulating metabolic pathways might aid in regulating stem cell fate between self-renewal and differentiation. Although the importance of AMPK in stem cells has not yet been extensively studied,it has been shown that AMPK activation inhibits mesenchymal stem cells proliferation[95] and fosters the differentiation of endothelial cells progenitors[96].

Very recently, Rodger etal. highlighted the capacity of quiescentmuscle stem cells to enter cell cycle intoa " $\mathrm{G}_{\text {Alert }}$ " phase $\left(\mathrm{G}_{0}\right.$-to- $\mathrm{G}_{1}$ pre-transition) [97]. " $\mathrm{G}_{\text {Alert" }}$ " transition of quiescent stem cellsallows a rapid response to injury,by priming the cells for cell cycle entry. This transition into the alert state is induced bythe activation of mechanistictarget of rapamycin complex $1(\mathrm{mTORC} 1)$ signaling, and by increases in mitochondrial number and function. Because mTORC1 and AMPK are two distinct and opposite signalingpathways[98100], the contribution of the opposing functions of mTORC1 and AMPK in this adaptive response to skeletal muscle injuryremains to be explored. 


\section{Skeletal muscle plasticity as a therapeutic target}

-Myokines and whole-body adaptations to exercise

Muscle contraction has emerged as an important activator of the synthesis and the release of severalsecretory cytokines and other peptidemolecules, known as myokines, that can exert either autocrine, paracrine, or endocrine effects. These myokines have been proposed to mediate whole-body adaptations to exercise through the regulation of skeletal muscle metabolism and signaling to distant metabolic organs. The cytokine interleukin-6 (IL)6 has been recognized as the prototypicalmyokine, contributing to muscle metabolic adaptation and the beneficial systemic effects of exercise training [101]. In the past decade, an abundance of evidence has emerged demonstrating that IL-6 stimulates glucose transport and fatty acid oxidation in skeletal muscle in an AMPK-dependent fashion [102]. Other myokines, such as IL-8 and IL-15, have also been shown more recently to increase glucose transport through an increase in AMPK phosphorylation [103]. Similarly, irisin, a newly discovered myokine mediating the effects of exercise on adipocyte browning, exerts a direct action onskeletal muscle metabolism through AMPK activation[104]. Interestingly, it appears that skeletal muscle irisin expression is controlled by an AMPK-PGC- $1 \alpha$ signaling cascade, suggesting the potential existence of a positive feedback loop [105]. AMPK activation has also been proposed to be involved in the mechanism governing IL-6 production and release. However, although AMPK was shown to regulate IL-6 release from isolated skeletal muscle in response to pharmacological activators of AMPK [106], recent evidence indicates that AMPK $\alpha 2$ is dispensable for contraction-stimulated IL-6 release [107].

\section{- Engineering skeletal muscle for repair}


Degenerating myopathies are characterized by chronic inflammation associated with permanent attempts atregeneration and persistent inflammation. Chronic activation of AMPK in the dystrophic mdx mouse, a murine model of Duchenne myopathy, with AICAR, improves the metabolic function of the skeletal muscle (mainlymitochondrial activity) and leads to an improvement of the histological pattern of the muscle by a reduction of both sarcolemma damage and regenerating events [108]. Besides the role of AMPK in myofiber and mitochondrial metabolism, AMPK also seems to act on the regulation of inflammation in the myopathic muscle. Fibrosis andIL-6 and IL-10 levels are reduced in the muscle of AICARtreated mdx mice [109]. Therefore, knowledge of the molecular regulation ofthe macrophage inflammatory profile may be useful in understanding howthe inflammation balance is disrupted in these diseases. In fibrotic mdx muscle, the myeloid population encompasses variousmacrophages subsets [110]. The balance between these various populations is currently unknown, but analysis of the involvement of AMPK in the balance between the macrophage subsets in fibrotic mdx may be relevant to improvingmyopathic muscle. Thus, novel knowledge of the mechanisms of skeletal muscle regeneration obtained through satellite cells and non-satellite cells holds great therapeutic potential for the treatment of muscle disorders.

\section{- Towards exercise mimetics: myth or reality?}

Physiological adaptations in skeletal muscle are widely recognized to improve wholebody performance and metabolism in health and disease. Several epidemiological and interventional studies have demonstrated that regular physical exercise is of great benefit in both the treatment and prevention of clinical conditions including obesity and type 2 diabetes. By contrast, physical inactivity is a recognized risk factor for these diseases. Several studies have reported an increased proportion of type IIb fibers and decreased numbers of type I 
fibers with obesity and insulin resistance[111, 112]. However, endurance exercise has been reported to cause a switch away from glycolytic type IIb fibers towards more oxidative type I and IIa fibers, and is therefore likely to be beneficial[113].In addition, regular moderateintensity exercise induces favorable changes in glucose intolerance and decreases mean plasma glucose levels in type 2 diabetic patients through increased muscle glucose disposal [114]. Thus, beyond human performance, there is great interest in promoting regular exercise, combined with an improved diet, not only for protection against the development of obesity and type 2 diabetes, but also as a first line of treatment[115].

Building on the observation that metabolic reprogramming, which favors oxidative over glycolytic metabolism, has a beneficial effect on skeletal muscle, several AMPK activators have been proposed as endurance exercise mimetics. For example, resveratrol, a natural polyphenolic compound mainly found in red wine and an AMPK activator, increases oxidative metabolism and endurance capacity in mice [116]. The effects of resveratrol are mediated by AMPK,as skeletal muscle mitochondrial biogenesis and endurance performance fail to increase in AMPK-deficient mice [117]. Similarly, AICAR also promotes a highendurance phenotype in mice[118]. The question remains as to what extend these data can be extrapolated to humans. The first clues will probably come from drug abuse by athletes, as AICAR has been recently added to the prohibited list of the World Anti-Doping Agency [119].

\section{Concluding remarks}

Recent major scientific advances have begun to unravel the signaling pathways as well as the genetic,biochemical, and metabolic properties ofskeletal muscle through which the beneficial effects of physical exercise arise. Studies have highlighted the potential of AMPKas an important integrator of cell signaling pathways that mediate the phenotypic 
plasticity of skeletal muscle, but some specific questions have not been fully explored(Box 2). AMPK activation is triggered by sustained contraction, and appears to be particularly important in the metabolic changes that occur during physical exercise. Mice lacking AMPK $\alpha$ or AMPK $\beta$ subunits in skeletal muscle have a dramatically impaired ability to perform skeletal muscle contractions and forced treadmill running, and also display reduced voluntary wheel running[35, 36]. Exercise-induced AMPK activation acutely activates glucose uptake by promoting translocation of the transporter GLUT4 to the membrane. While further studies are warranted to fully understand the mechanism by which AMPK promotes GLUT4 translocation, likely candidates are TBC1D1 and TCB1D4 [28], which have been proposed to be the converging point with the insulin signaling pathway and endothelial Nitric Oxide Synthase (eNOS), which generates nitric oxide in blood vessels and is involved in regulating vascular tone[71]. Understanding how AMPK and other signals are integrated to elicit specific changes in glucose transport depending on muscle fiber type and exercise intensity remains a challenge that the field still needs to address. Another important metabolic pathway targeted by AMPK during contraction is fatty acid oxidation. Recent studies from musclespecific AMPK $\alpha 1 \alpha 2$-deficient micedemonstrated the essential role ofAMPK in enhancing fatty acid utilization during exercise. Prolonged AMPK activation also causes some of the chronic adaptations to endurance exercise, such as increased GLUT4 expression and mitochondrial biogenesis, through the modulation of PGC-1 $\alpha$ and SIRT1 activities.Lastly, AMPK appears to bea crucial contributor to phagocytosis-induced macrophage skewing during the regeneration of damaged skeletal muscle.

Given the various degrees of beneficial phenotypic plasticity demonstrated by chronic AMPK stimulation in pre-clinical animal models, the potential of pharmacological activation of AMPK in humans represents a significant challenge. A better understanding of the molecular mechanisms involved in the control of skeletal muscle plasticity is important for the opening 
of new avenues for pharmacological targets in type 2 diabetes and obesity as well as in muscle disorders such as muscular dystrophy. Interestingly, changes in the AMPK heterotrimer composition and AMPK activation are attenuated with age in response to endurance-type muscle contraction and may contribute to skeletal muscle metabolic dysfunction in aged sarcopenic muscle $[68,120]$. However, reductions in skeletal muscle AMPK activity are not sufficient to promote the development of aging-induced insulinresistance [121],indicating that AMPK activation may lead to improved strategies for preventingthe reduced mitochondrial function and dysregulated intracellular lipid metabolism in skeletal muscle associated with aging. 


\section{Box 1: Skeletal muscle regeneration}

Skeletal muscle regeneration relies on resident adult stem cells, named satellite cells,thatundertake a series of cell-fate decisions to ensure efficient repair of the damaged muscle fibers. Briefly, these cells remain quiescent under the basal lamina of myofibers and are activated after injury. Once activated, these precursor muscle cells proliferate, differentiate and fuse to reform new myofibers. A small subset of these precursor muscle cells do not differentiate, and return into quiescence to reform a sufficient pool of muscle stem cells for further needs [86]. However, to be complete, skeletal muscle regeneration also needs the involvement of other cells such as perivascular cells and immune cells like macrophages[79].After injury, circulating monocytes enter into the damaged muscle and differentiate into pro-inflammatory (M1) macrophages. These M1 macrophages secrete proinflammatory cytokines, phagocytose debris/necrotic myofibers, and activate muscle precursor cell proliferation. These macrophages then skew their phenotype and became antiinflammatory (M2) macrophages[77]. At this time, M2 macrophages secrete antiinflammatory cytokines and sustain myogenesis; that is, the differentiation and fusion of muscle precursor cells[77].

\section{Box 2: Outstanding questions}

- What is the role of AMPK and its variouscomplexes in mediating glycolytic-to-oxidative fiber-type transformation?

- What is the specific mechanism by which AMPK promotes GLUT4 translocation during exercise?

- Does AMPK regulate muscle stem cell-fate choice by way of a "metabolic switch"?

- How is AMPK involved in the balance between different types of macrophages in dystrophic muscle? 


\section{Figure legends}

Figure 1:Schematic representation of AMP-activated protein kinase(AMPK)subunit isoforms.

AMPK is a heterotrimeric complex comprising a catalytic subunit $(\alpha)$ and two regulatory subunits $(\beta$ and $\gamma)$, existing as multiple isoforms $(\alpha 1, \alpha 2, \beta 1, \beta 2, \gamma 1, \gamma 2$, and $\gamma 3)$. AMPK $\alpha$ contains a kinase domain phosphorylated by upstream kinases on the residue Thr172, an autoinhibition domain (AID), and a domain that interacts with the $\beta$ subunits $[\beta$ subunit interacting domain( $\beta$-ID)]. AMPK $\beta$ subunit presents domains that interact with glycogen

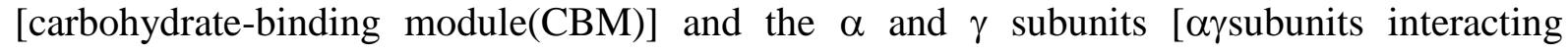
domain( $\alpha \gamma$-ID)]. AMPK $\gamma$ contains cystathionine $\beta$-synthase (CBS) domains binding AMP, ADP, and ATP, but only three of the four potential nucleotide-binding sites contribute to nucleotide regulation, with CBS-2 always unoccupied, CBS-4 permanently bound toAMP and CBS-1 and -3 binding AMP, ADP, and ATP interchangeably.

Figure 2: Regulation of AMP-activated protein kinase (AMPK)by exercise.

Following skeletal muscle contraction, AMP and ADP cellular concentrations and $\mathrm{Ca}^{2+}$ levels increase. Binding of ADP and AMP to the $\gamma$ subunit of AMPK triggers Thr172 phosphorylation by the upstream kinase liver kinase B1 (LKB1) protects against dephosphorylation, maintaining the kinase in an active conformation. After exercise, AMPK is returned to an inactive form by dephosphorylation catalyzed by the action of protein phosphatases (PP) 1A, 2A, and2C).

Figure 3:AMP-activated protein kinase (AMPK) heterotrimer composition in skeletal muscle. 
A cartoon of the predominant AMPK heterotrimers in human (quadriceps), murine glycolytic (EDL, extensor digitorum longus) and oxidative (soleus) muscles is shown.

Figure 4:Schematic overview of AMPK targets in the regulation of skeletal muscle adaptation to exercise

Following exercise, AMPK is activated by low energy status and regulates glucose uptake (TBC1D1/ TBC1D4), fatty acid uptake (TBC1D1), fatty acid oxidation (ACC2), mitochondrial biogenesis (PGC-1 $\alpha$ ), mitophagy (ULK1), and gene transcription (HDAC5, PGC-1 $\alpha$, FoxO3).

Key to abbreviations: ACC, acetyl CoA carboxylase; FoxO3, Forkhead box protein 3; HDAC5, histone deacetylase 5; PGC-1 $\alpha$, peroxisome proliferator-activated receptor gamma coactivator-1 $\alpha$; SIRT1, silent mating-type information regulator 2 homolog 1; TBC1D1/4, Tre-2/BUB2/cdc domain family domain family, member1/4; ULK1, uncoordinated-51-like kinase 1.

\section{Figure 5: Roles of AMPK in skeletal muscle regeneration.}

After an injury of skeletal muscle by cardiotoxin injection, muscle stem cells activate, proliferate and enter into the terminal differentiation program to fuse and to form new myofibers. A small subset of muscle cells do not differentiate but return to quiescence to reform a pool of muscle stem cells (i.e. self-renewal). AMPK implication in these steps remains unknown. In parallel, monocyte/macrophages infiltrate the place of injury, acquire pro-inflammatory (M1) phenotype and promote myoblast proliferation. Then, proinflammatory (M1) macrophages skew into anti-inflammatory (M2) macrophages, by an activation of the CaMKK $\beta / A M P K$ pathway, linked to the phagocytosis of apoptotic/necrotic 
myofibers. These anti-inflammatory (M2) macrophages sustain myoblast differentiation and fusion [84].

Key to abbreviations: CTX: cardiotoxin, Mo : monocytes, MPs : macrophages

\section{Acknowledgements}

The work of B.V.'s group is performed within Département Hospitalo-Universitaire (DHU) AUToimmune and HORmonal diseaseS and was supported by grants from the INSERM, the CNRS, the Université Paris Descartes, the Agence Nationale de la Recherche, the Association Française contre les Myopathies, and aEuropean Commission integrated project (LSHM-CT2004-005272/ exgenesis).The work of R.M.'s group is supported by grants from the INSERM, the CNRS, the Université Claude Bernard Lyon1, theAssociation Française contre les Myopathies, the Ligue Nationale Contre le Cancer, the Agence Française de Lutte Contre le Dopage,and the Société Française de Myologie.

The authors have no conflict of interest related to this work. 


\section{References}

1 Bassel-Duby, R. and Olson, E.N. (2006) Signaling pathways in skeletal muscle remodeling. Annu Rev Biochem 75, 19-37

2 Fluck, M. (2006) Functional, structural and molecular plasticity of mammalian skeletal muscle in response to exercise stimuli. J Exp Biol 209, 2239-2248

3 Hood, D.A., et al. (2006) Coordination of metabolic plasticity in skeletal muscle. J Exp Biol 209, 2265-2275

4 Hardie, D.G. (2014) AMPK-Sensing Energy while Talking to Other Signaling Pathways. Cell Metab 20, 939-952

5 Jensen, T.E., et al. (2009) AMP-activated protein kinase in contraction regulation of skeletal muscle metabolism: necessary and/or sufficient? Acta Physiol (Oxf) 196, 155-174

6 Jensen, T.E. and Richter, E.A. (2012) Regulation of glucose and glycogen metabolism during and after exercise. J Physiol 590, 1069-1076

7 O'Neill, H.M., et al. (2013) AMPK regulation of fatty acid metabolism and mitochondrial biogenesis: implications for obesity. Mol Cell Endocrinol 366, 135-151

8 McGee, S.L. and Hargreaves, M. (2011) Histone modifications and exercise adaptations. $J$ Appl Physiol (1985) 110, 258-263

9 McBride, A., et al. (2009) The glycogen-binding domain on the AMPK beta subunit allows the kinase to act as a glycogen sensor. Cell Metab 9, 23-34

10 Wojtaszewski, J.F., et al. (2003) Regulation of 5'AMP-activated protein kinase activity and substrate utilization in exercising human skeletal muscle. Am J Physiol Endocrinol Metab 284, E813-822

11 Sakamoto, K., et al. (2005) Deficiency of LKB1 in skeletal muscle prevents AMPK activation and glucose uptake during contraction. Embo J 24, 1810-1820

12 Thomson, D.M., et al. (2007) Skeletal muscle and heart LKB1 deficiency causes decreased voluntary running and reduced muscle mitochondrial marker enzyme expression in mice. $A m$ J Physiol Endocrinol Metab 292, E196-202

13 Oakhill, J.S., et al. (2011) AMPK is a direct adenylate charge-regulated protein kinase. Science 332, 1433-1435

14 Xiao, B., et al. (2011) Structure of mammalian AMPK and its regulation by ADP. Nature 472, 230-233

15 Cheung, P.C., et al. (2000) Characterization of AMP-activated protein kinase gammasubunit isoforms and their role in AMP binding. Biochem J $346 \mathrm{Pt} \mathrm{3,659-669}$

16 Suter, M., et al. (2006) Dissecting the role of 5'-AMP for allosteric stimulation, activation, and deactivation of AMP-activated protein kinase. The Journal of biological chemistry 281, 32207-32216

17 Hawley, S.A., et al. (2005) Calmodulin-dependent protein kinase kinase-beta is an alternative upstream kinase for AMP-activated protein kinase. Cell Metab 2, 9-19

18 Woods, A., et al. (2005) Ca2+/calmodulin-dependent protein kinase kinase-beta acts upstream of AMP-activated protein kinase in mammalian cells. Cell Metab 2, 21-33

19 Jensen, T.E., et al. (2007) Possible CaMKK-dependent regulation of AMPK phosphorylation and glucose uptake at the onset of mild tetanic skeletal muscle contraction. Am J Physiol Endocrinol Metab 292, E1308-1317

20 McGee, S.L., et al. (2008) Normal hypertrophy accompanied by phosphoryation and activation of AMP-activated protein kinase alpha1 following overload in LKB1 knockout mice. J Physiol 586, 1731-1741

21 Birk, J.B. and Wojtaszewski, J.F. (2006) Predominant alpha2/beta2/gamma3 AMPK activation during exercise in human skeletal muscle. $J$ Physiol 577, 1021-1032 
22 Treebak, J.T., et al. (2009) A-769662 activates AMPK beta1-containing complexes but induces glucose uptake through a PI3-kinase-dependent pathway in mouse skeletal muscle. Am J Physiol Cell Physiol 297, C1041-1052

23 Barnes, B.R., et al. (2004) The 5'-AMP-activated protein kinase gamma3 isoform has a key role in carbohydrate and lipid metabolism in glycolytic skeletal muscle. The Journal of biological chemistry 279, 38441-38447

24 Milan, D., et al. (2000) A mutation in PRKAG3 associated with excess glycogen content in pig skeletal muscle. Science 288, 1248-1251

25 Costford, S.R., et al. (2007) Gain-of-function R225W mutation in human AMPKgamma(3) causing increased glycogen and decreased triglyceride in skeletal muscle. PLoS One 2, e903

26 Treebak, J.T., et al. (2007) AS160 phosphorylation is associated with activation of alpha2beta2gamma1- but not alpha2beta2gamma3-AMPK trimeric complex in skeletal muscle during exercise in humans. Am J Physiol Endocrinol Metab 292, E715-722

27 McGee, S.L., et al. (2003) Exercise increases nuclear AMPK alpha2 in human skeletal muscle. Diabetes 52, 926-928

28 Treebak, J.T., et al. (2014) Acute exercise and physiological insulin induce distinct phosphorylation signatures on TBC1D1 and TBC1D4 proteins in human skeletal muscle. $J$ Physiol 592, 351-375

29 Warden, S.M., et al. (2001) Post-translational modifications of the beta-1 subunit of AMPactivated protein kinase affect enzyme activity and cellular localization. Biochem J 354, 275283

30 Pinter, K., et al. (2013) Localisation of AMPK gamma subunits in cardiac and skeletal muscles. J Muscle Res Cell Motil 34, 369-378

31 Rockl, K.S., et al. (2007) Skeletal muscle adaptation to exercise training: AMP-activated protein kinase mediates muscle fiber type shift. Diabetes 56, 2062-2069

32 Suwa, M., et al. (2003) Effects of chronic AICAR treatment on fiber composition, enzyme activity, UCP3, and PGC-1 in rat muscles. J Appl Physiol (1985) 95, 960-968

33 Reyes, N.L., et al. (2014) Fnip1 regulates skeletal muscle fiber type specification, fatigue resistance, and susceptibility to muscular dystrophy. Proc Natl Acad Sci U S A

34 Garcia-Roves, P.M., et al. (2008) Gain-of-function R225Q mutation in AMP-activated protein kinase gamma3 subunit increases mitochondrial biogenesis in glycolytic skeletal muscle. The Journal of biological chemistry 283, 35724-35734

35 O'Neill, H.M., et al. (2011) AMP-activated protein kinase (AMPK) beta1beta2 muscle null mice reveal an essential role for AMPK in maintaining mitochondrial content and glucose uptake during exercise. Proc Natl Acad Sci U S A 108, 16092-16097

36 Lantier, L., et al. (2014) AMPK controls exercise endurance, mitochondrial oxidative capacity, and skeletal muscle integrity. Faseb J 28, 3211-3224

37 Merrill, G.F., et al. (1997) AICA riboside increases AMP-activated protein kinase, fatty acid oxidation, and glucose uptake in rat muscle. Am J Physiol 273, E1107-1112

38 Winder, W.W. and Hardie, D.G. (1996) Inactivation of acetyl-CoA carboxylase and activation of AMP-activated protein kinase in muscle during exercise. Am J Physiol 270, E299-304

39 Hutber, C.A., et al. (1997) Electrical stimulation inactivates muscle acetyl-CoA carboxylase and increases AMP-activated protein kinase. Am J Physiol 272, E262-266

40 Vavvas, D., et al. (1997) Contraction-induced changes in acetyl-CoA carboxylase and 5'AMP-activated kinase in skeletal muscle. The Journal of biological chemistry 272, 1325513261

41 O'Neill, H.M., et al. (2014) AMPK phosphorylation of ACC2 is required for skeletal muscle fatty acid oxidation and insulin sensitivity in mice. Diabetologia 57, 1693-1702 
42 Roepstorff, C., et al. (2005) Malonyl-CoA and carnitine in regulation of fat oxidation in human skeletal muscle during exercise. Am J Physiol Endocrinol Metab 288, E133-142

43 Miura, S., et al. (2009) Alpha2-AMPK activity is not essential for an increase in fatty acid oxidation during low-intensity exercise. Am J Physiol Endocrinol Metab 296, E47-55

44 Dzamko, N., et al. (2008) AMPK-independent pathways regulate skeletal muscle fatty acid oxidation. J Physiol 586, 5819-5831

45 Jeppesen, J., et al. (2013) LKB1 regulates lipid oxidation during exercise independently of AMPK. Diabetes 62, 1490-1499

46 Jeppesen, J., et al. (2011) Contraction-induced skeletal muscle FAT/CD36 trafficking and FA uptake is AMPK independent. J Lipid Res 52, 699-711

47 Fentz, J., et al. (2015) AMPKalpha is critical for enhancing skeletal muscle fatty acid utilization during in vivo exercise in mice. FASEB J. http://dx.doi.org/10.1096/fj.14-266650 Published online January 21, 2015

48 Richter, E.A. and Hargreaves, M. (2013) Exercise, GLUT4, and skeletal muscle glucose uptake. Physiol Rev 93, 993-1017

49 Szekeres, F., et al. (2012) The Rab-GTPase-activating protein TBC1D1 regulates skeletal muscle glucose metabolism. Am J Physiol Endocrinol Metab 303, E524-533

50 Chadt, A., et al. (2014) Deletion of Both Rab-GTPase-Activating Proteins TBC1D1 and TBC1D4 in Mice Eliminates Insulin- and AICAR-Stimulated Glucose Transport. Diabetes 51 Stockli, J., et al. (2015) The RabGAP TBC1D1 plays a central role in exercise-regulated glucose metabolism in skeletal muscle. Diabeteshttp://dx.doi.org/10.2337/db13-1489 Published online January 9, 2015

52 Musi, N., et al. (2001) AMP-activated protein kinase (AMPK) is activated in muscle of subjects with type 2 diabetes during exercise. Diabetes 50, 921-927

53 Musi, N., et al. (2002) Metformin increases AMP-activated protein kinase activity in skeletal muscle of subjects with type 2 diabetes. Diabetes 51, 2074-2081

54 Hayashi, T., et al. (1998) Evidence for 5' AMP-activated protein kinase mediation of the effect of muscle contraction on glucose transport. Diabetes 47, 1369-1373

55 Jorgensen, S.B., et al. (2004) Knockout of the alpha2 but not alpha1 5'-AMP-activated protein kinase isoform abolishes 5-aminoimidazole-4-carboxamide-1-beta-4ribofuranosidebut not contraction-induced glucose uptake in skeletal muscle. The Journal of biological chemistry 279, 1070-1079

56 Lai, Y.C., et al. (2014) A small-molecule benzimidazole derivative that potently activates AMPK to increase glucose transport in skeletal muscle: comparison with effects of contraction and other AMPK activators. Biochem J 460, 363-375

57 Pehmoller, C., et al. (2009) Genetic disruption of AMPK signaling abolishes both contraction- and insulin-stimulated TBC1D1 phosphorylation and 14-3-3 binding in mouse skeletal muscle. Am J Physiol Endocrinol Metab 297, E665-675

58 Treebak, J.T., et al. (2006) AMPK-mediated AS160 phosphorylation in skeletal muscle is dependent on AMPK catalytic and regulatory subunits. Diabetes 55, 2051-2058

59 Wright, D.C., et al. (2005) Contraction- and hypoxia-stimulated glucose transport is mediated by a Ca2+-dependent mechanism in slow-twitch rat soleus muscle. Am J Physiol Endocrinol Metab 288, E1062-1066

60 Jensen, T.E., et al. (2014) Contraction-stimulated glucose transport in muscle is controlled by AMPK and mechanical stress but not sarcoplasmatic reticulum $\mathrm{Ca}(2+)$ release. Mol Metab 3, 742-753

61 McGee, S.L. and Hargreaves, M. (2010) AMPK-mediated regulation of transcription in skeletal muscle. Clin Sci (Lond) 118, 507-518

62 Jager, S., et al. (2007) AMP-activated protein kinase (AMPK) action in skeletal muscle via direct phosphorylation of PGC-1alpha. Proc Natl Acad Sci U S A 104, 12017-12022 
63 Canto, C., et al. (2010) Interdependence of AMPK and SIRT1 for metabolic adaptation to fasting and exercise in skeletal muscle. Cell Metab 11, 213-219

64 McKinsey, T.A., et al. (2000) Signal-dependent nuclear export of a histone deacetylase regulates muscle differentiation. Nature 408, 106-111

65 McGee, S.L., et al. (2014) Compensatory regulation of HDAC5 in muscle maintains metabolic adaptive responses and metabolism in response to energetic stress. FASEB journal 28, 3384-3395

66 Leick, L., et al. (2008) PGC-1alpha is not mandatory for exercise- and training-induced adaptive gene responses in mouse skeletal muscle. Am J Physiol Endocrinol Metab 294, E463-474

67 Zong, H., et al. (2002) AMP kinase is required for mitochondrial biogenesis in skeletal muscle in response to chronic energy deprivation. Proc Natl Acad Sci U S A 99, 15983-15987 68 Reznick, R.M., et al. (2007) Aging-associated reductions in AMP-activated protein kinase activity and mitochondrial biogenesis. Cell Metab 5, 151-156

69 Tanner, C.B., et al. (2013) Mitochondrial and performance adaptations to exercise training in mice lacking skeletal muscle LKB1. Am J Physiol Endocrinol Metab 305, E1018-1029

70 Winder, W.W., et al. (2000) Activation of AMP-activated protein kinase increases mitochondrial enzymes in skeletal muscle. J Appl Physiol (1985) 88, 2219-2226

71 Lee-Young, R.S., et al. (2009) Skeletal muscle AMP-activated protein kinase is essential for the metabolic response to exercise in vivo. The Journal of biological chemistry 284, 23925-23934

$72 \mathrm{Mu}$, J., et al. (2001) A role for AMP-activated protein kinase in contraction- and hypoxiaregulated glucose transport in skeletal muscle. Mol Cell 7, 1085-1094

73 Grumati, P., et al. (2011) Physical exercise stimulates autophagy in normal skeletal muscles but is detrimental for collagen VI-deficient muscles. Autophagy 7, 1415-1423

$74 \mathrm{He}, \mathrm{C}$., et al. (2012) Exercise-induced BCL2-regulated autophagy is required for muscle glucose homeostasis. Nature 481, 511-515

75 Sanchez, A.M., et al. (2012) AMPK promotes skeletal muscle autophagy through activation of forkhead FoxO3a and interaction with Ulk1. J Cell Biochem 113, 695-710

76 Lo Verso, F., et al. (2014) Autophagy is not required to sustain exercise and PRKAA1/AMPK activity but is important to prevent mitochondrial damage during physical activity. Autophagy 10, 1883-1894

77 Saclier, M., et al. (2013) Monocyte/macrophage interactions with myogenic precursor cells during skeletal muscle regeneration. Febs $J$ 280, 4118-4130

78 Saclier, M., et al. (2013) Differentially activated macrophages orchestrate myogenic precursor cell fate during human skeletal muscle regeneration. Stem Cells 31, 384-396

79 Arnold, L., et al. (2007) Inflammatory monocytes recruited after skeletal muscle injury switch into antiinflammatory macrophages to support myogenesis. The Journal of experimental medicine 204, 1057-1069

80 Jeong, H.W., et al. (2009) Berberine suppresses proinflammatory responses through AMPK activation in macrophages. Am J Physiol Endocrinol Metab 296, E955-964

81 Sag, D., et al. (2008) Adenosine 5'-monophosphate-activated protein kinase promotes macrophage polarization to an anti-inflammatory functional phenotype. J Immunol 181, 86338641

82 Zhao, X., et al. (2008) Activation of AMPK attenuates neutrophil proinflammatory activity and decreases the severity of acute lung injury. Am J Physiol Lung Cell Mol Physiol 295, L497-504

83 Xing, J., et al. (2013) Inhibition of AMP-activated protein kinase accentuates lipopolysaccharide-induced lung endothelial barrier dysfunction and lung injury in vivo. Am J Pathol 182, 1021-1030 
84 Mounier, R., et al. (2013) AMPKalpha1 Regulates Macrophage Skewing at the Time of Resolution of Inflammation during Skeletal Muscle Regeneration. Cell Metab 18, 251-264

85 Visvader, J.E. and Lindeman, G.J. (2012) Cancer stem cells: current status and evolving complexities. Cell Stem Cell 10, 717-728

86 Yin, H., et al. (2013) Satellite cells and the muscle stem cell niche. Physiol Rev 93, 23-67

87 Shan, T., et al. (2014) Lkb1 is indispensable for skeletal muscle development, regeneration, and satellite cell homeostasis. Stem Cells 32, 2893-2907

88 Gopinath, S.D., et al. (2014) FOXO3 promotes quiescence in adult muscle stem cells during the process of self-renewal. Stem Cell Reports 2, 414-426

89 Christov, C., et al. (2007) Muscle satellite cells and endothelial cells: close neighbors and privileged partners. Mol Biol Cell 18, 1397-1409

90 Abou-Khalil, R., et al. (2009) Autocrine and paracrine angiopoietin 1/Tie-2 signaling promotes muscle satellite cell self-renewal. Cell Stem Cell 5, 298-309

91 Ylikorkala, A., et al. (2001) Vascular abnormalities and deregulation of VEGF in Lkb1deficient mice. Science 293, 1323-1326

92 Simsek, T., et al. (2010) The distinct metabolic profile of hematopoietic stem cells reflects their location in a hypoxic niche. Cell Stem Cell 7, 380-390

93 Rafalski, V.A., et al. (2012) Energy metabolism and energy-sensing pathways in mammalian embryonic and adult stem cell fate. J Cell Sci 125, 5597-5608

94 Ryall, J.G., et al. (2015) The NAD(+)-Dependent SIRT1 Deacetylase Translates a Metabolic Switch into Regulatory Epigenetics in Skeletal Muscle Stem Cells. Cell Stem Cell $16,171-183$

95 de Meester, C., et al. (2014) Role of AMP-activated protein kinase in regulating hypoxic survival and proliferation of mesenchymal stem cells. Cardiovasc Res 101, 20-29

96 Zhu, Z., et al. (2011) Prostaglandin E2 promotes endothelial differentiation from bone marrow-derived cells through AMPK activation. PLoS One 6, e23554

97 Rodgers, J.T., et al. (2014) mTORC1 controls the adaptive transition of quiescent stem cells from G0 to G(Alert). Nature 510, 393-396

98 Mounier, R., et al. (2009) Important role for AMPKalpha1 in limiting skeletal muscle cell hypertrophy. Faseb J 23, 2264-2273

99 Lantier, L., et al. (2010) Coordinated maintenance of muscle cell size control by AMPactivated protein kinase. Faseb $J$ 24, 3555-3561

100 Mounier, R., et al. (2011) Antagonistic control of muscle cell size by AMPK and mTORC1. Cell Cycle 10, 2640-2646

101 Pedersen, B.K. and Febbraio, M.A. (2012) Muscles, exercise and obesity: skeletal muscle as a secretory organ. Nat Rev Endocrinol 8, 457-465

102 Carey, A.L., et al. (2006) Interleukin-6 increases insulin-stimulated glucose disposal in humans and glucose uptake and fatty acid oxidation in vitro via AMP-activated protein kinase. Diabetes 55, 2688-2697

103 Gray, S.R. and Kamolrat, T. (2011) The effect of exercise induced cytokines on insulin stimulated glucose transport in C2C12 cells. Cytokine 55, 221-228

104 Huh, J.Y., et al. (2014) Exercise-induced irisin secretion is independent of age or fitness level and increased irisin may directly modulate muscle metabolism through AMPK activation. J Clin Endocrinol Metab 99, E2154-2161

105 Shan, T., et al. (2013) Myostatin knockout drives browning of white adipose tissue through activating the AMPK-PGC1alpha-Fndc5 pathway in muscle. Faseb J 27, 1981-1989

106 Glund, S., et al. (2009) Role of adenosine 5'-monophosphate-activated protein kinase in interleukin-6 release from isolated mouse skeletal muscle. Endocrinology 150, 600-606

107 Lauritzen, H.P., et al. (2013) Contraction and AICAR stimulate IL-6 vesicle depletion from skeletal muscle fibers in vivo. Diabetes 62, 3081-3092 
108 Ljubicic, V. and Jasmin, B.J. (2013) AMP-activated protein kinase at the nexus of therapeutic skeletal muscle plasticity in Duchenne muscular dystrophy. Trends Mol Med 19, 614-624

109 Jahnke, V.E., et al. (2012) Metabolic remodeling agents show beneficial effects in the dystrophin-deficient mdx mouse model. Skelet Muscle 2, 16

110 Desguerre, I., et al. (2012) A new model of experimental fibrosis in hindlimb skeletal muscle of adult mdx mouse mimicking muscular dystrophy. Muscle Nerve 45, 803-814

111 Wade, A.J., et al. (1990) Muscle fibre type and aetiology of obesity. Lancet 335, 805-808 112 Kriketos, A.D., et al. (1997) Muscle fibre type composition in infant and adult populations and relationships with obesity. Int J Obes Relat Metab Disord 21, 796-801

113 Thayer, R., et al. (2000) A decade of aerobic endurance training: histological evidence for fibre type transformation. J Sports Med Phys Fitness 40, 284-289

114 Goodyear, L.J. and Kahn, B.B. (1998) Exercise, glucose transport, and insulin sensitivity. Annu Rev Med 49, 235-261

115 Albright, A., et al. (2000) American College of Sports Medicine position stand. Exercise and type 2 diabetes. Med Sci Sports Exerc 32, 1345-1360

116 Lagouge, M., et al. (2006) Resveratrol improves mitochondrial function and protects against metabolic disease by activating SIRT1 and PGC-1alpha. Cell 127, 1109-1122

$117 \mathrm{Um}, \mathrm{J} . \mathrm{H}$., et al. (2010) AMP-activated protein kinase-deficient mice are resistant to the metabolic effects of resveratrol. Diabetes 59, 554-563

118 Narkar, V.A., et al. (2008) AMPK and PPARdelta agonists are exercise mimetics. Cell $134,405-415$

119 Pokrywka, A., et al. (2014) Metabolic modulators of the exercise response: doping control analysis of an agonist of the peroxisome proliferator-activated receptor delta (GW501516) and 5-aminoimidazole-4-carboxamide ribonucleotide (AICAR). J Physiol Pharmacol 65, 469-476

120 Hardman, S.E., et al. (2014) The effects of age and muscle contraction on AMPK activity and heterotrimer composition. Exp Gerontol 55, 120-128

121 Bujak, A.L., et al. (2014) Reduced skeletal muscle AMPK and mitochondrial markers do not promote age-induced insulin resistance. J Appl Physiol (1985) 117, 171-179 
Figure 1

\begin{tabular}{|c|c|c|c|c|}
\hline \multirow[b]{2}{*}{$\alpha 1 \mathrm{~N}$} & upst & 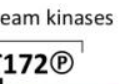 & & $\beta$ binding \\
\hline & kinase & domain & AID & $\beta-I D$ \\
\hline$\alpha 2 \mathrm{~N}-$ & kinase & domain & AID & $\beta-I D$ \\
\hline
\end{tabular}

\begin{tabular}{|c|c|c|}
\hline & glycogen binding & $\alpha$ and $\gamma$ binding \\
\hline$\beta 1 \mathrm{~N}-$ & CBM & $\alpha \gamma-1 D$ \\
\hline$B 2 \mathrm{~N}-$ & CBM & $\alpha \gamma-I D$ \\
\hline
\end{tabular}

\begin{tabular}{|c|c|c|}
\hline \multirow[b]{2}{*}{ ү1 } & $\beta$ binding & AMP/ADP/ATP binding \\
\hline & $\mathrm{N}-$ & CBS1-CBS2-CBS3-CBS4 - \\
\hline 2 & & CBS1-CBS2-CBS3-CBS4 - \\
\hline 3 & $\mathrm{~N}-1$ & CBS1-CBS2-CBS3-CBS4 - \\
\hline & & $\begin{array}{ll}\text { ATP } & \text { ATP AMP } \\
\text { ADP } & \text { ADP } \\
\text { AMP } & \text { AMP }\end{array}$ \\
\hline
\end{tabular}

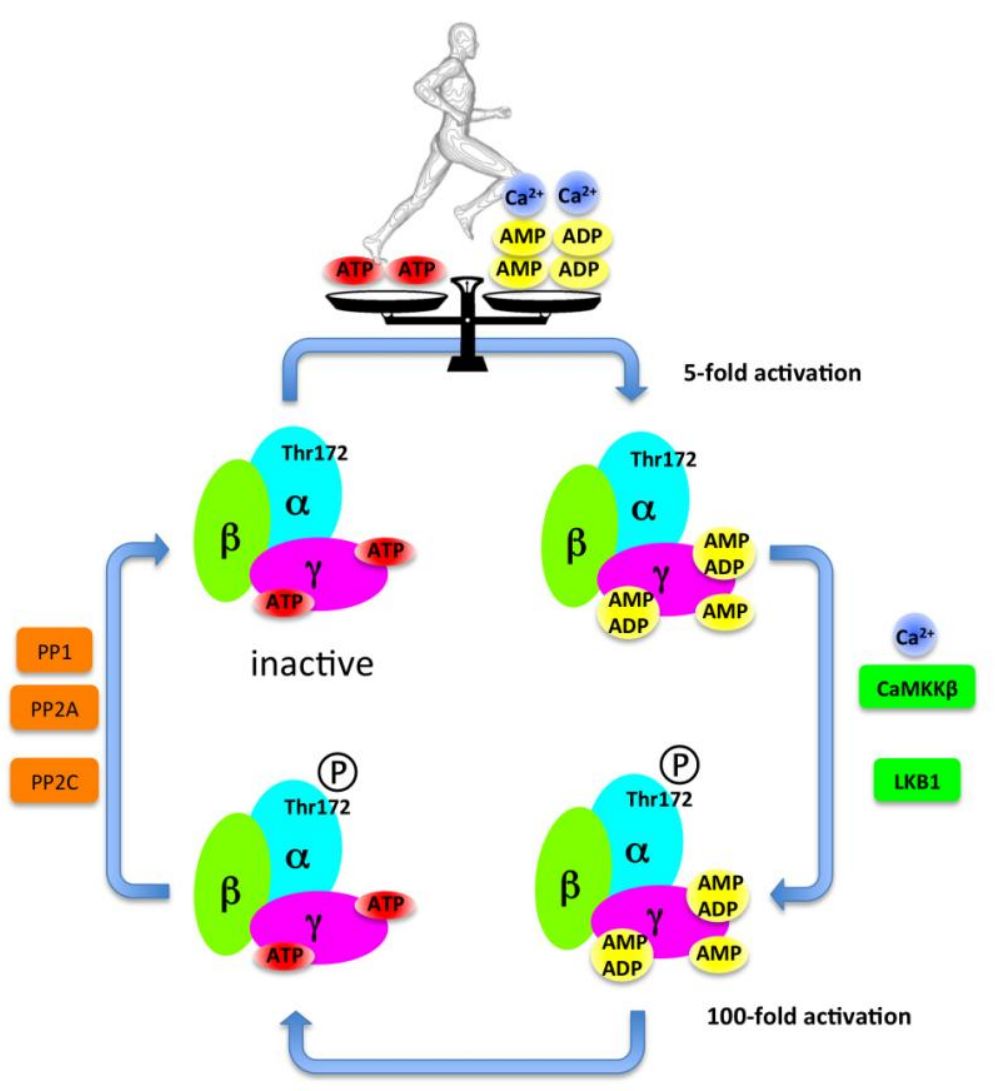

Figure 2 
Figure 3
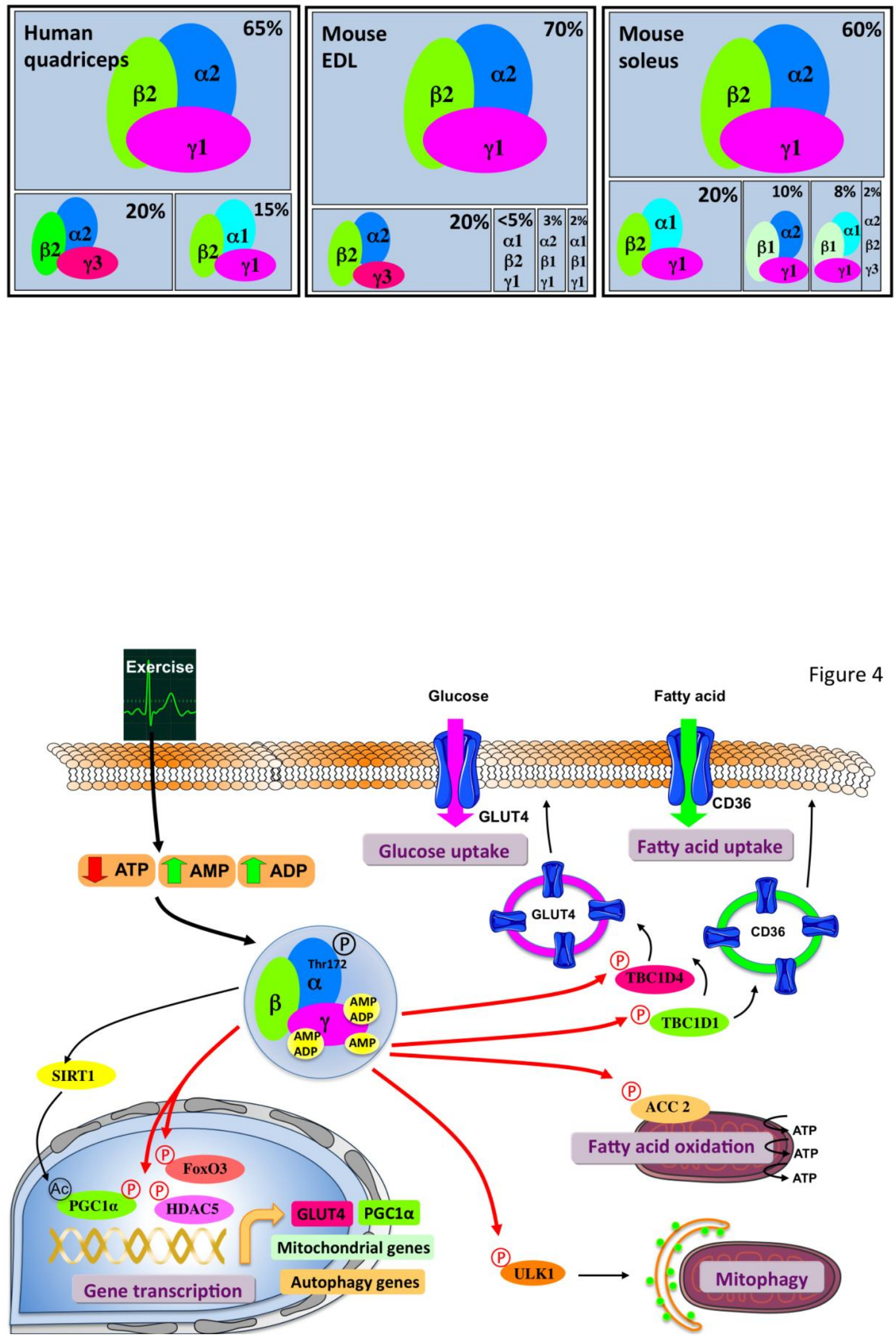


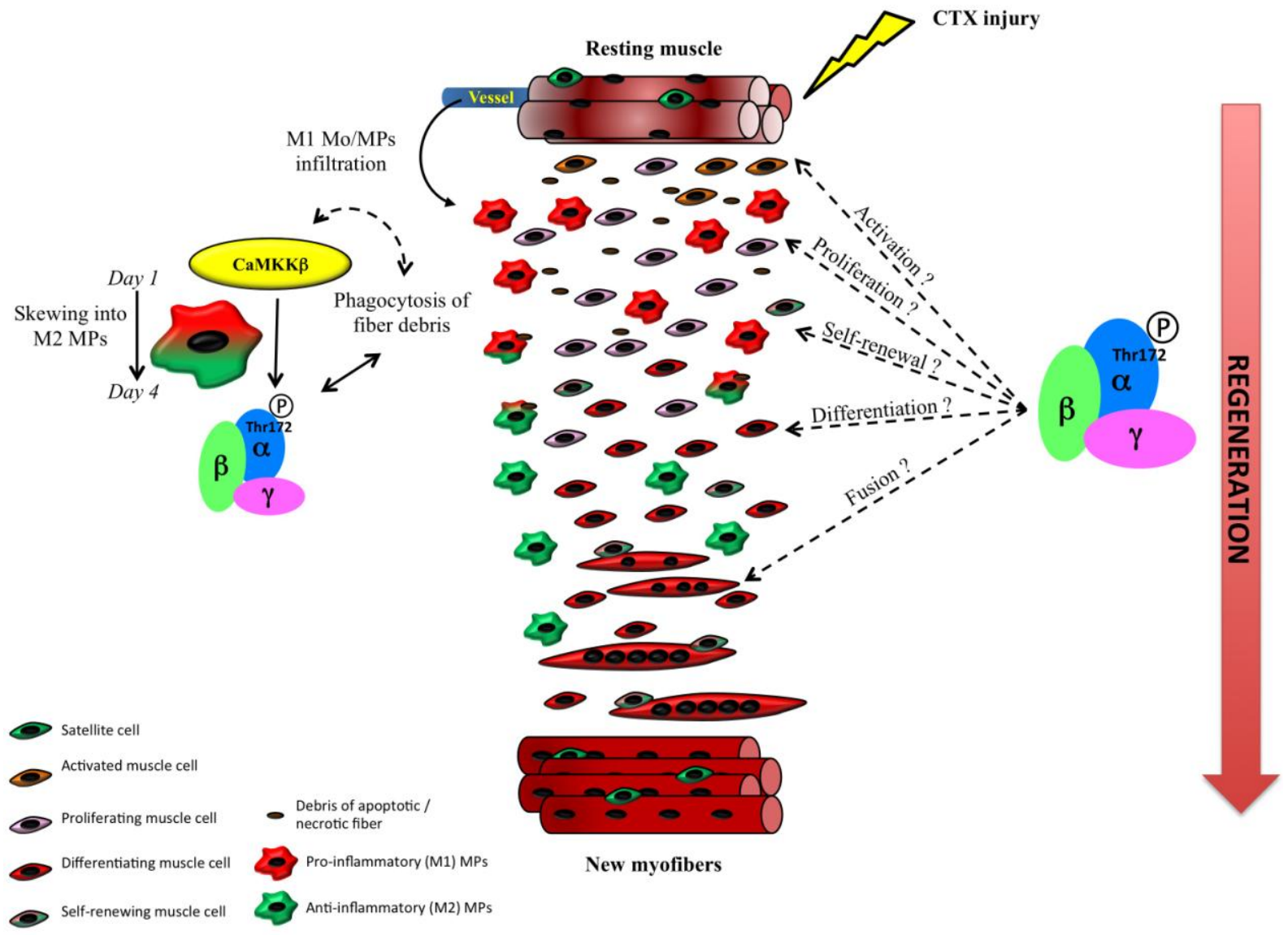

